\title{
The Magnetization and Curie Temperature of the Double Exchange Interaction Systems
}

\author{
Chong Der Hu \\ Department of Physics, National Taiwan University, Taipei, Taiwan R.O.C.
}

(Received June 9, 2000)

\begin{abstract}
We studied the double exchange interaction. Starting from [Kubo and Ohata's Hamiltonian: J. Phys. Soc. Jpn. 33 (1972) 21] we used a 1/(2S+1) expansion to calculate the thermodynamic potential, magnetization and $T_{\mathrm{C}}$. It was found that including superexchange interaction results in realistic Curie temperature.
\end{abstract}

KEYWORDS: colossal magnetoresistance, Curie temperature, magnetization, manganite

\section{$\S 1 . \quad$ Introduction}

Doped manganese oxides (typically $\mathrm{La}_{1-x} \mathrm{D}_{x} \mathrm{MnO}_{3}$ where $\mathrm{D}$ is a divalent element) have exhibited many facinating physical properties, most notably is the coupling between magnetism and transport properties. ${ }^{1)}$ In the doping range $0.2 \leq x \leq 0.5$, these compounds are ferromagnetic metals. For smaller doping and doping range $x \geq 0.5$, the manganites are antiferromagnetic insulators. As temperature rises, they become paramagnetic insulators. They also show very large (negative) magnetoresistance (CMR). These properties promoted Zener ${ }^{2)}$ to propose the double exchange (DE) mechanism for explanation. In this model, a $\mathrm{Mn}^{+3}$ and a $\mathrm{Mn}^{+4}$ ion can exchange electrons through the oxygen ion in the middle. According to the Hund's rules, the spins of the exchanged electrons must be parallel to the spins of manganese ions in order to have lower energy. Therefore it is advantageous to have ferromagnetic states so that electrons have extended wave functions and lower kinetic energy. Recently, Millis et al. called attention to the lattice distortion ${ }^{3,4)}$ and some experiments showed that physical properties such as magnetization and resistivty are related to structure transition. ${ }^{5-7)}$ They argued that the only relevant energy scale is the hopping energy of electrons $t / S$. Estimation of the spin wave energy based on DE gave approximately $x z t / S$ where $x$ is the doping concentration and $z$ is the coordination number. Since band structure calculation ${ }^{8)}$ gave $t \simeq 0.2 \mathrm{eV}$, the Curie temperature was in the range of $2400^{\circ} \mathrm{K}$ at $x=1 / 3$. This is almost ten times greater than experiments results, thus raised the question of the role DE played in manganites. On the other hand, Furukawa calculated $T_{\mathrm{C}}$ with the infinite-dimension Kondo-Lattice model ${ }^{9)}$ and concluded that $T_{\mathrm{C}}$ was in the range of $200^{\circ} \mathrm{K}$. Varma ${ }^{10)}$ and Sheng et $a l .{ }^{11,12)}$ used the localization picture and obtained $T_{\mathrm{C}}$ in the similar region. The situation needs to be clarified. However, most recent theoretical works on the CMR systems considered more than just DE. Coulomb interactions, Yahn-Teller distortion, superexchange interaction and orbital degeneracy were incorporated. While these interactions do exist in the manganites and may be nec- essary to obtain realistic results, including them renders the system overly complicated and results difficult to analyze. If DE is the fundamental mechanism in manganites (at least in the region $x \leq 0.5$ ), then it is of vital importance to solve it, or at least find a controlled perturbation. Calculations, including other interactions mentioned above, should be based on the picture of DE to avoid unexpected error. Therefore, we studied DE in details. Our goal is o examine DE to see whether it can produce reasonable Curie temperature and other physical properties and provide a basic picture so that based on which futher calculation can proceed.

Important progress on studying DE was first made by Anderson and Hasegawa ${ }^{13}$ ) who simplified it to a oneband model and wrote down explicitly the relation between local spins and electron motion. De Gennes ${ }^{14)}$ made the approximation of treating the local spins classically and incoporated superexchange interaction. Similar approaches were still widely used recently. Kubo and Ohata ${ }^{15)}$ made next important contribution by introducing projection operators so as to consider only those states having lowest energy according to Hund's rules. Recent analytical study of DE includes Furukawa's works on the infinite-dimension Kondo lattice model, ${ }^{9}$ ) the Schwinger boson approach of Sarker, ${ }^{16)}$ CPA method by Edwards et al. ${ }^{17)}$ and Okabe's study of spin waves and lowly excitations. ${ }^{18)}$ We developed a perturbative approach, the $1 /(2 S+1)$ series expansion, combined with a finite temperature technique for spin operators. We calculated the magnetization and Curie temperature. In $\S 2$, we gave an introduction to the Hamiltonian. We derive the $1 /(2 S+1)$ series expansion in $\S 3$. In $\S 4$, we presented the calculation of the thermodynamic potential. The resulting magnetization and Curie temperature along with discussion were shown in $\S 5$.

\section{§2. Hamiltonian}

The double exchange interaction has two essential elements, namely the hopping of conduction electrons and exchange interaction between local spins and conduction electrons. 


$$
H=-t \sum_{i, j, \sigma} c_{i \sigma}^{\dagger} c_{j \sigma}-I \sum_{i} \boldsymbol{S}_{i} \cdot \boldsymbol{\sigma}_{i}
$$

where $i$ and $j$ are nearest neighbors and $\boldsymbol{\sigma}$ is the electron spin. In the perovskite manganites $I \gg t$. In fact $I$ is greater than the average kinetic energy $x z t$. Therefore it is a good approximation to assume that the electron spin is always parallel to the local spin. Based on the deduction, Kubo and Ohata ${ }^{15)}$ simplified above Hamiltonian by project out only those states having the lowest energy according to the Hund's rules. This approach has the advantage of treating the local spin quantum mechanically. Their Hamiltonian for holes is

$$
H=-t \sum_{i, j, \alpha, \beta} c_{i \alpha}^{\dagger}\left(P_{i} P_{j}\right)_{\alpha \beta} c_{j \beta},
$$

where the projection operator $P$ is used to projects out the states in which the spins of holes anti-parallel to the local spins:

$$
P_{i}=\frac{S-\boldsymbol{S}_{i} \cdot \boldsymbol{\sigma}}{2 S+1}
$$

The Hamiltonian can be expanded as

$$
\begin{aligned}
H= & -t \sum_{i, j \alpha, \beta} c_{i \alpha}^{\dagger}\left[S^{2}+\boldsymbol{S}_{i} \cdot \boldsymbol{S}_{j}\right. \\
& -\left(S S_{i z}+S S_{j z}+\frac{1}{2} S_{i-} S_{j+}-\frac{1}{2} S_{i+} S_{j-}\right) \sigma_{z} \\
& -\left(S S_{i-}+S S_{j-}-S_{i z} S_{j-}+S_{i-} S_{j z}\right) \frac{\sigma_{-}}{2} \\
& \left.-\left(S S_{i+}+S S_{j+}+S_{i z} S_{j+}-S_{i+} S_{j z}\right) \frac{\sigma_{+}}{2}\right]_{\alpha \beta} c_{j \beta} .
\end{aligned}
$$

We can divide eq. (4) into a mean-field part $\left(H_{0}\right)$ and fluctuation parts $\left(\mathrm{H}_{1-4}\right)$

$$
H=H_{0}+H_{1}+H_{2}+H_{3}+H_{4}
$$

where

$$
\begin{aligned}
& H_{0}=-\frac{t}{N(2 S+1)^{2}} \sum_{i, j, \boldsymbol{k}, \sigma} \mathrm{e}^{\mathrm{i} \boldsymbol{k} \cdot \boldsymbol{R}_{i j}}\left[\left(S-\sigma\left\langle S_{z}\right\rangle\right)^{2} c_{\boldsymbol{k}, \sigma}^{\dagger} c_{\boldsymbol{k}, \sigma}+\left(S_{i z}+S_{j z}-2\left\langle S_{z}\right\rangle\right)\left(\left\langle S_{z}\right\rangle-\sigma S\right) n_{\boldsymbol{k}, \sigma}\right], \\
& H_{1}=-\frac{t}{N(2 S+1)^{2}} \sum_{i, j, \boldsymbol{k}, \sigma} \mathrm{e}^{\mathrm{i} \boldsymbol{k} \cdot \boldsymbol{R}_{i j}+\mathrm{i} \boldsymbol{q} \cdot \boldsymbol{R}_{i}}\left(c_{\boldsymbol{k}-\boldsymbol{q}, \sigma}^{\dagger} c_{\boldsymbol{k}, \sigma}-n_{\boldsymbol{k}, \sigma}\right)\left(S_{i z}+S_{j z}-2\left\langle S_{z}\right\rangle\right)\left(\left\langle S_{z}\right\rangle-\sigma S\right), \\
& H_{2}=-\frac{t}{N(2 S+1)^{2}} \sum_{i, j, \boldsymbol{k}, \boldsymbol{q}, \sigma} \mathrm{e}^{\mathrm{i} \boldsymbol{k} \cdot \boldsymbol{R}_{i j}+\mathrm{i} \boldsymbol{q} \cdot \boldsymbol{R}_{i}} c_{\boldsymbol{k}-\boldsymbol{q}, \sigma}^{\dagger} c_{\boldsymbol{k}, \sigma}\left(S_{i z}-\left\langle S_{z}\right\rangle\right)\left(S_{j z}-\left\langle S_{z}\right\rangle\right), \\
& H_{3}=-\frac{t}{N(2 S+1)^{2}} \sum_{i, j, \boldsymbol{k}, \boldsymbol{q}} \mathrm{e}^{\mathrm{i} \boldsymbol{k} \cdot \boldsymbol{R}_{i j}+\mathrm{i} \boldsymbol{q} \cdot \boldsymbol{R}_{i}\left(c_{\boldsymbol{k}-\boldsymbol{q}, \uparrow}^{\dagger} c_{\boldsymbol{k}, \uparrow} S_{i-} S_{j+}+c_{\boldsymbol{k}-\boldsymbol{q}, \downarrow}^{\dagger} c_{\boldsymbol{k}, \downarrow} S_{i+} S_{j-}\right)}
\end{aligned}
$$

and

$$
\begin{aligned}
H_{4}= & -\frac{t}{N(2 S+1)^{2}} \sum_{i, j, \boldsymbol{k}, \boldsymbol{q}} \mathrm{e}^{\mathrm{i} \boldsymbol{k} \cdot \boldsymbol{R}_{i j}+\mathrm{i} \boldsymbol{q} \cdot \boldsymbol{R}_{i}}\left[c_{\boldsymbol{k}-\boldsymbol{q}, \uparrow}^{\dagger} c_{\boldsymbol{k}, \downarrow}\left(S_{i z} S_{i-}-S_{i-} S_{j z}-S S_{i-}-S S_{j-}\right)\right. \\
& \left.+c_{\boldsymbol{k}-\boldsymbol{q}, \downarrow}^{\dagger} c_{\boldsymbol{k}, \uparrow}\left(-S S_{i+}-S S_{j+}+S_{i+} S_{j z}-S_{i z} S_{j+}\right)\right]
\end{aligned}
$$

where $\langle X\rangle$ is the thermal average and $n_{\boldsymbol{k} \sigma}=\left\langle c_{\boldsymbol{k} \sigma}^{\dagger} c_{\boldsymbol{k} \sigma}\right\rangle$. In our mathematical expression $\sigma=1(-1)$ for spin-up (down) holes. $H_{1}$ and $H_{2}$ describe the scattering of holes by the $S_{z}$ fluctuations and $H_{3}$ and $H_{4}$ the spin-flip scattering.

$H_{0}$ contains the most important contribution. The energy of a hole with spin polarization $\sigma$ is

$$
\varepsilon_{\boldsymbol{k}, \sigma}=-\left(S-\sigma\left\langle S_{z}\right\rangle\right)^{2} \varepsilon_{\boldsymbol{k}},
$$

where

$$
\varepsilon_{\boldsymbol{k}}=\frac{t}{(2 S+1)^{2}} \sum_{n . n .} \mathrm{e}^{\mathrm{i} \boldsymbol{k} \cdot \boldsymbol{R}},
$$

and $\sum_{n . n}$. sums over nearest neighbors. The spin-up and spin-down holes form two bands. Their levels and shapes are related to $\left\langle S_{z}\right\rangle$. Since for $\left\langle S_{z}\right\rangle>0$, spin-down holes has a wider and lower band, one easily deduce they are the majority and more mobile. As temperature rises, two bands approach each other. The spin-down holes become more massive and fewer in number and the spin-up holes follow the opposite trend.

$H_{0}$ also contains the dominant magnetic mechanism. Due to the second term in eq. (6), the energy level spac- ing of local spins is

$$
\omega_{0}=2 \sum_{\boldsymbol{k}, \sigma}\left(\left\langle S_{z}\right\rangle-\sigma S\right) f_{\boldsymbol{k} \sigma} \varepsilon_{\boldsymbol{k}}
$$

where $f_{\boldsymbol{k} \sigma}$ is the Fermi-Dirac distribution function of holes with spin polarization $\sigma$. In the summantion, $\sigma$ is taken to be \pm 1 . We see that $\omega_{0}$ is affected by the abundance of holes of both spin-orientations. As temperature rises, $\omega_{0}$ decreases because of the decrease of both $\left\langle S_{z}\right\rangle$ and the difference beteween the spin-up and spin-down hole densities. At the level of $H_{0}$, the local spins are independent of each other. Hence,

$$
\left\langle S_{z}\right\rangle=S B_{S}\left(S \beta \omega_{0}\right),
$$

where $B_{S}\left(S \beta \omega_{0}\right)$ is the Brillouin function. However, since $\left\langle S_{z}\right\rangle$ affects the energy bands of holes and hence, the hole density in each spin orientation, it must be solved self-consistently.

To reduce the amount of computation, we used a free hole band in place of that in eq. (12).

$$
\varepsilon_{\boldsymbol{k}}=\frac{t\left(6.5 k^{2}-z\right)}{(2 S+1)^{2}}
$$


where $k$ is the crystal momuntum in unit of $\pi / a$. Since the number of spin-up (down) holes is related to $\left\langle S_{z}\right\rangle$ through energy, and it will affect the double-exchange coupling, we make sure that the bottoms of the spin-up free hole band and the spin-down one are the same as those in eq. (11), and thus have approximately the correct number of holes of either spin polarization. Next, since we are interested in the situations where doping is less than 0.5, only the lower-half of the band is of importance. So we simulated the lower-half band by making the density of states of the free hole band similar to that in eq. (11). Thus we do not expect this approximation to produce serious error. Most importantly, it will give correct qualitative behaviors.

In order to compare our results with experiments, we added a superexchange (SE) term to the Hamiltonian

$$
H_{S E}=\frac{J}{2} \sum_{i, j} \boldsymbol{S}_{i} \cdot \boldsymbol{S}_{j} .
$$

SE is rather weak. We have $J=t^{2} / U$ where $U$ is the on-site Coulomb repulsion. Since $U$ is of the order of $6 \mathrm{eV}$, we choose $J=t / 30$. It will be considered in a similar fashion as DE. The part $z J \sum S_{i z}\left\langle S_{z}\right\rangle$ willl be incoporated in the mean-field level, such that eq. (13) will be modified as

$$
\omega_{0}=2 \sum_{\boldsymbol{k}, \sigma}\left(\left\langle S_{z}\right\rangle-\sigma S\right) f_{\boldsymbol{k} \sigma} \varepsilon_{\boldsymbol{k}}+z J\left\langle S_{z}\right\rangle .
$$

Its fluctuation contribution will be considered in $\S 4$. Because $J$ is rather small, we do not expect any serious error.

\section{§3. $1 /(2 S+1)$ Expansion}

We intended to study the finite-temperature properties. Therefore, we cannot use any approximation, such as Houlstein-Primakoff transformation, which is suited only for very low temperatures. Fortunately, there exists $1 /(2 S+1)$ expansion for temperature below $T_{\mathrm{C}}$. We show that having extracted the mean-field part $H_{0}$ from the Hamiltonian, the fluctuation part can be treated perturbatively. We also discuss in what temperature range the expansion is applicable.

The $n$-th power of $H_{1}$ or $H_{2}$ are, at most, proportional to

$$
\frac{(2 S)^{n} \prod_{l}\left\langle\left(S_{z}-\left\langle S_{z}\right\rangle\right)^{l}\right\rangle^{n_{l}}}{(2 S+1)^{2 n}}, \quad \sum_{l} n_{l} l=n .
$$

Here, $n$ is the number of hoppings and $l$ is the number of hopping onto a certain site. It was shown that $\left\langle\left(S_{z}-\right.\right.$ $\left.\left.\left\langle S_{z}\right\rangle\right)^{l}\right\rangle$ can be written as linear combinations of Brillouin function and its derivatives. ${ }^{19)}$ For example,

$$
\begin{aligned}
\left\langle S_{z}\right\rangle & =S B_{S}\left(S \beta \omega_{0}\right) \\
& =\left(S+\frac{1}{2}\right) \operatorname{coth} \frac{(2 S+1) \beta \omega_{0}}{2}-\frac{1}{2} \operatorname{coth} \frac{\beta \omega_{0}}{2}
\end{aligned}
$$

and

$$
\begin{aligned}
\left\langle S_{z}^{2}\right\rangle-\left\langle S_{z}\right\rangle^{2} & =-\left.S^{2} \frac{\mathrm{d} B_{S}(x)}{\mathrm{d} x}\right|_{x=S \beta \omega_{0}} \\
& =\left(S+\frac{1}{2}\right)^{2} \sinh ^{-2} \frac{(2 S+1) \beta \omega_{0}}{2}
\end{aligned}
$$

$$
-\frac{1}{4} \sinh ^{-2} \frac{\beta \omega_{0}}{2} .
$$

For $(S+1 / 2) \beta \omega_{0}>1$, we have

$$
\begin{aligned}
\left\langle S_{z}\right\rangle \simeq & \left(S+\frac{1}{2}\right)\left\{1+2 \exp \left[-(2 S+1) \beta \omega_{0}\right]\right\} \\
& -\frac{1}{2} \operatorname{coth} \frac{\beta \omega_{0}}{2}
\end{aligned}
$$

and

$$
\begin{aligned}
\left\langle S_{z}^{2}\right\rangle-\left\langle S_{z}\right\rangle^{2} \simeq & (2 S+1)^{2} \exp \left[-(2 S+1) \beta \omega_{0}\right] \\
& -\frac{1}{4} \sinh ^{-2} \frac{\beta \omega_{0}}{2} .
\end{aligned}
$$

For the $1 /(2 S+1)$ expansion to be valid, we should stay in the temperature range

$$
\mathrm{e}^{(2 S+1) \beta \omega_{0}}>2 S+1
$$

which is approximately

$$
(S+1 / 2) \beta \omega_{0}>1,
$$

for $S=2$. In this range, $\left\langle\left(S_{z}-\left\langle S_{z}\right\rangle\right)^{l=2}\right\rangle$ and will be reduced by at least a factor of $2 S+1$. For $l>2$, the situation is similar because the Brillouin function and all its derivatives are reduced by at least a factor of $2 S+1$. As a result, (17) is reduced by a factor of $(2 S+1)^{m}$ where $m$ is the number of nonzero $n_{l}$ 's. It would seem that the largest contribution comes from the term $n_{1}+2 n_{2}=$ $n$ and $n_{l \neq 1,2}=0 .{ }^{20)}$ This is not the case. Because in this term, all the $S_{z}$ must be at the same two sites and so do the hoppings of holes. Hence, its magnitude is reduces by afoctor of $z^{n-2}$. Thus, the magnitudes of higher order terms are reduced by two factors. If the hoppings concentrate at a few sites, the kinetic energy of holes will be smaller. On the other hand, if holes have extensive motion, then fluctuations occur at many sites. Each one will produce a factor of $1 /(2 S+1)$. In either case, the higher order terms are small and and the perturbative series of $H_{1}$ are convergent, and likewise for $\mathrm{H}_{2}$.

During the expansion, we are going to have terms like $\left\langle S_{+} S_{-} . . S_{z} S_{z} \ldots\right\rangle$ if $H_{3}$ or $H_{4}$ are involved. Each pair of $S_{+} S_{-}$can be reduced to $S_{z}$ or $\left(S_{z}\right)^{0}$ (see ref. 19 or appendix for examples). Hence, whenever a term contains a pair of $S_{+} S_{-}$, its power of $S$ is reduced by one. $H_{3}$ and $H_{4}$ can also be treated perturbatively.

The "radius of convergence" shown in (20a) and (20b) is in fact quite extended. In view of eq. (13) we found that $\omega_{0}$ is temperature-dependent. In Fig. 1 we show $\omega_{0}$ from $H_{0}$ versus temperature for $x=1 / 3$. It was found that $(20)$ 's and the $1 /(2 S+1)$ expansion are valid if $T<0.9 T_{\mathrm{C}}$.

\section{§4. Thermodynamic Potential}

To find the magnetization and the Curie temperature of the system, it is natural to calculate the thermodynamic potential. We consider the $1 /(2 S+1)$ expansion. The term from $H_{0}$ is easily done,

$$
\Omega_{0}=-k_{\mathrm{B}} T \sum_{\boldsymbol{k}, \boldsymbol{\sigma}} \ln \left[1+\mathrm{e}^{-\beta\left(\varepsilon_{k \sigma}-\mu\right)}\right]
$$




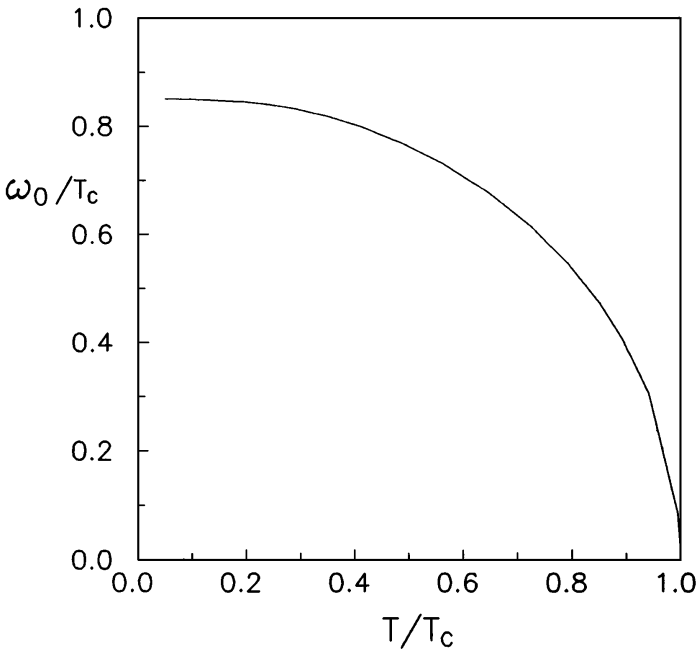

Fig. 1. $\omega_{0} / T_{\mathrm{C}}$ versus $T / T_{\mathrm{C}}$ at $x=1 / 3$.

$$
-N k_{\mathrm{B}} T \ln \frac{\sinh \left[(S+1 / 2) \beta \omega_{0}\right]}{\sinh \left(\beta \omega_{0} / 2\right)}+N\left\langle S_{z}\right\rangle \omega_{0}
$$

We also get the spin waves propagator from $H_{0}$ :

$$
\begin{aligned}
D_{0}\left(\mathrm{i} q_{n}\right) & =-\frac{1}{2} \int_{-\beta}^{\beta} \mathrm{d} \tau\left\langle\hat{T} S_{+}(\tau) S_{-}\left(\tau^{\prime}\right)\right\rangle \mathrm{e}^{\mathrm{i} q_{n}\left(\tau-\tau^{\prime}\right)} \\
& =\frac{2\left\langle S_{z}\right\rangle}{\mathrm{i} q_{n}-\omega_{0}} .
\end{aligned}
$$

The next order terms (of the order $(2 S+1)^{-1}$ ), are $\Omega_{2 f}$ and $\Omega_{2 z}$ which come from $H_{4}$ and $H_{1}$ respectively.

$$
\Omega_{2 f}=-\frac{k_{\mathrm{B}} T}{2} \sum_{\boldsymbol{q}, n} \cdot \chi_{t}\left(\boldsymbol{q}, q_{n}, \sigma\right) \frac{2\left\langle S_{z}\right\rangle}{-\mathrm{i} q_{n} \sigma-\omega_{0}},
$$

and

$$
\Omega_{2 z}=-\frac{1}{2} \sum_{\boldsymbol{q}, n} \cdot \chi_{z}\left(\boldsymbol{q}, q_{n}, \sigma\right)\left[\left\langle S_{z}^{2}\right\rangle-\left\langle S_{z}\right\rangle^{2}\right],
$$

where

$$
\begin{aligned}
& \chi_{t}\left(\mathrm{i} q_{n}, \boldsymbol{q}, \sigma\right) \\
& =\frac{1}{N} \sum_{\boldsymbol{p}}\left[\left(S-\sigma\left\langle S_{z}\right\rangle\right) \varepsilon_{\boldsymbol{p}}+\left(S+\sigma\left\langle S_{z}\right\rangle\right) \varepsilon_{\boldsymbol{p}-\boldsymbol{q}}\right]^{2} \\
& \quad \times \frac{f_{\boldsymbol{p}-\boldsymbol{q},-\sigma}-f_{\boldsymbol{p}, \sigma}}{\mathrm{i} q_{n}-\varepsilon_{\boldsymbol{p}, \sigma}+\varepsilon_{\boldsymbol{p}-\boldsymbol{q},-\sigma}}
\end{aligned}
$$

and

$$
\begin{aligned}
\chi_{z}\left(\mathrm{i} q_{n}, \boldsymbol{q}, \sigma\right)= & \frac{1}{N} \sum_{\boldsymbol{p}}\left(S-\sigma\left\langle S_{z}\right\rangle\right)^{2}\left(\varepsilon_{\boldsymbol{p}}+\varepsilon_{\boldsymbol{p}-\boldsymbol{q}}\right)^{2} \\
& \times \frac{f_{\boldsymbol{p}-\boldsymbol{q}, \sigma}-f_{\boldsymbol{p}, \sigma}}{\mathrm{i} q_{n}-\varepsilon_{\boldsymbol{p}, \sigma}+\varepsilon_{\boldsymbol{p}-\boldsymbol{q}, \sigma}}
\end{aligned}
$$

$\chi_{t}$ and $\chi_{z}$ have the similar structure. Both contain hole polarizations and the square of couplings. The difference is that the former comes from the spin-flip process and the latter the fluctuations of $S_{z}$. Accordingly, they are respective sources of $\Omega_{2 f}$ and $\Omega_{2 z}$. We shall only consider above two kinds of fluctuations in a infinite series and neglect other fluctuations which are at least of the or$\operatorname{der}(2 S+1)^{-2}$. Having performed the coupling-constant integration, we get the thermodynamic potential

$$
\begin{aligned}
\Omega= & \Omega_{0}+\frac{k_{\mathrm{B}} T}{2} \sum_{\boldsymbol{q}, n, \sigma} \ln \left\{1-\chi_{t}\left(\boldsymbol{q}, q_{n}, \sigma\right) \frac{2\left\langle S_{z}\right\rangle}{-\mathrm{i} q_{n} \sigma-\omega_{0}}-\beta \chi_{z}\left(\boldsymbol{q}, q_{n}, \sigma\right)\left[\left\langle S_{z}^{2}\right\rangle-\left\langle S_{z}\right\rangle^{2}\right]\right\} \\
& +k_{\mathrm{B}} T \sum_{\boldsymbol{q}, n} \ln \left[1-\frac{\left\langle S_{z}\right\rangle J F(\boldsymbol{q})}{\mathrm{i} q_{n}-\omega_{0}}\right]+k_{\mathrm{B}} T \sum_{\boldsymbol{q}} \ln \left[1-\beta J F(\boldsymbol{q})\left\langle S_{z}^{2}-\left\langle S_{z}\right\rangle^{2}\right\rangle\right]
\end{aligned}
$$

where

$$
F(\boldsymbol{q})=\sum_{n . n .} \mathrm{e}^{\mathrm{i} \boldsymbol{q} \cdot \boldsymbol{r}}
$$

and the last two terms come from SE. ${ }^{21)} \mathrm{SE}$ and DE were considered on the same footing.

One can easily see that there two adjustable parameters, namely $\left\langle S_{z}\right\rangle$ and the chemical potential $\mu$. We can adjust $\mu$ so that the hole density is equal to the doping concentration. On the other hand, $\left\langle S_{z}\right\rangle$ is fixed selfconsistently. For a given $\left\langle S_{z}\right\rangle$, we can calculate $\Omega$ and then $\left\langle S_{z}\right\rangle$. The self-consistency is reached if $\left\langle S_{z}\right\rangle$ input is equal to $\left\langle S_{z}\right\rangle$ output. To calculate $\left\langle S_{z}\right\rangle$ from $\Omega$, we applied a fictitious magnetic field to the system which couples only to the local spins.

$$
H_{e x t}=-\sum_{i} q \mu_{\mathrm{B}} \boldsymbol{H} \cdot \boldsymbol{S}_{i}
$$

Taking the derivative of $\Omega$ with respect to $\boldsymbol{H}$ (pointing in the $z$-direction) gives $\left\langle S_{z}\right\rangle$. Note that the effect of $H_{\text {ext }}$ is simply changing $\omega_{0}$ into $\omega_{0}+g \mu_{\mathrm{B}}|\boldsymbol{H}|$, hence $\left\langle S_{z}\right\rangle$ is easily calculated. The magnetization can be found by adding to $\left\langle S_{z}\right\rangle$ the spins of holes. We present the result in next section.

\section{$\S 5 . \quad$ Results and Discussion}

The principle goal of this work is to see whether DE can give a reasonable Curie temperature, and hence, clarify the issue of whether DE is the fundamental mechanism in manganites. To this end, we calculated the magnetization $M(T)$ as a function of temperature. Though our calculation is not very accurate near $T_{\mathrm{C}}$, the error is not significant. This can be seen in Fig. 2 where we plotted $M(T)$ under the conditions $t=0.2 \mathrm{eV}$ and $x=1 / 3$ and $x=0.2$. It is found that $M(T)$ saturated as $T \rightarrow 0$ and decreased rapidly near $T_{\mathrm{C}}$. It is only in the latter region that our calculation is not very accurate. Since the temperature range of this region is small, the inaccuracy is not expected to create serious error in $T_{\mathrm{C}}$.

We presented the calculated Curie temperature versus doping concentration in Fig. 3. The solid and dashed curves are those calculated with and without superexchange interaction. Without $\mathrm{SE}, T_{\mathrm{C}}$ rises sharply with $x$. But it shows a tendency of saturation at $700^{\circ} \mathrm{K}$ as 


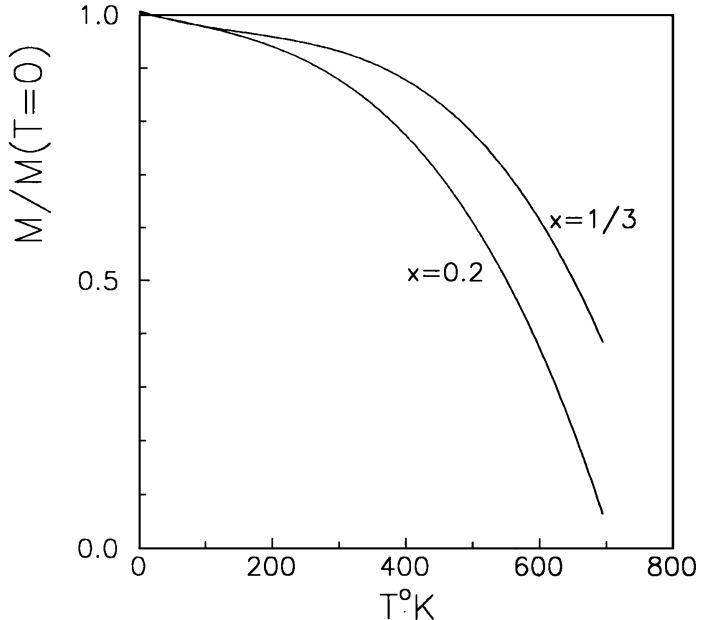

Fig. 2. Magnetizations (divided by their repective saturation values at $T=0$ ) at doping concentrations $x=0.2$ and $x=1 / 3$ are plotted against temperature.

$x \rightarrow 0.5$. Recall that a previous estimate of the spin wave energy in DE system gives $T_{\mathrm{C}} \simeq x z t / S \simeq 2400^{\circ} \mathrm{K}$. The reason for this discrepancy is that the estimation neglected the fact that as temperature increases, the spin wave softened. In other words, as the system moves toward $T_{\mathrm{C}}$, the spin-down holes become less numerous and the effective coupling between up-local spins becomes weaker. On the other hand, the coupling between downlocal spins becomes stronger. This effect is huge because it reduces $T_{\mathrm{C}}$ to one third of its original estimated value. It is also interesting to see that the $T_{\mathrm{C}}$ of a pure $\mathrm{DE}$ system does not varies as $x(1-x)$ as one would have assumed. There is some subtlety in DE. As we mentioned before, the spin-up hole band and the spin-down hole band are seperated. For small doping and low temperature, the chemical potential is below the bottom of the spin-up hole band. Hence, most holes have down spins. It takes a large amount of energy (relative to temperature) to convert a spin-down hole to a spin-up one. The conversion will not be feasible until temperature is raised so high that the band seperation is comparable to temperature. Since the spin-down holes couple only the up-local spins, the ferromagnetism is rigid and $T_{\mathrm{C}}$ drops slowly as $x \rightarrow 0$. As the doping increases, chemical potential rises and moves closer to the bottom of the spin-up band. Now spin-up holes become more energetically favorable. Increasing doping introduces not only more spin-down holes but also more spin-up ones. The spin-up ones favor down local-spins. This reduces the strength of ferromagnetism. So we have two factors giving opposite trends at large $x$. More holes means stronger ferromagnetic coupling and higher $T_{\mathrm{C}}$. But the band structure of hole favors ferromagnetism at small $x$ and antiferromagnetism at large $x$. As a result, $T_{\mathrm{C}}$ drops slowly at small $x$ and saturates at large $x$.

With the addition of SE, ferromagnetism is suppressed and $T_{\mathrm{C}}$ is comparable to experimental data. However, one would have needed an unphysically large SE if the estimation $T_{\mathrm{C}} \simeq x z t$ had been correct. At $x \simeq 0.012$, the ferromagnetism becomes unstable due the presence

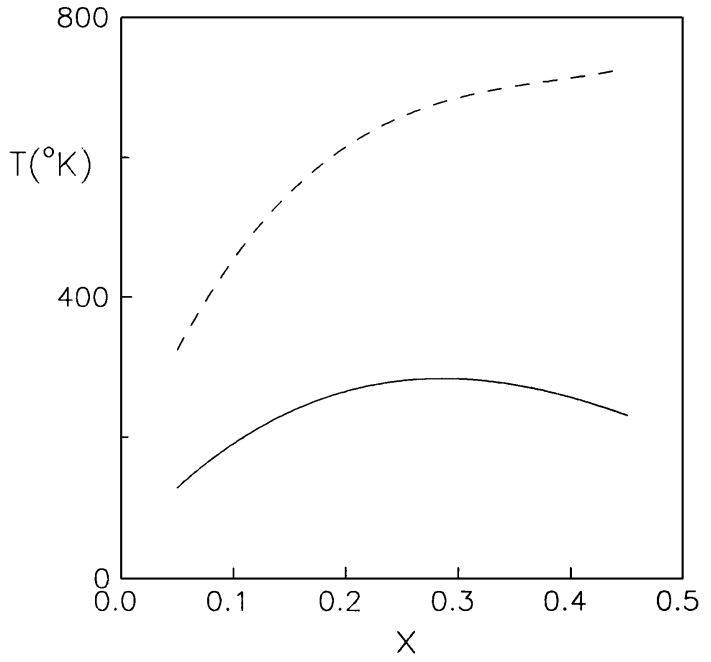

Fig. 3. Curie temperature versus doping concentration. The dashed line is the result of pure double exchange interaction and the solid line is that with the addition of superexchange interaction.

of SE. A interesting fact is that SE has a larger effect at large $x$. This again can be explained by the band structure. As we mentioned before at small doping the energies of spin-up and spin-down holes are farther apart. It is more difficult for SE to take effect. A system with large doping is not so rigid. The chemical potential approaches or even surpasses the bottom of the spin-up hole band, the conversion between spin-up holes and spindown holes are easier. With the addition of SE, the curve of $T_{\mathrm{C}}$ in Fig. 3 are compatible with the phase diagram given by experiments. We also remark that we did not attempt to find a best fit with $J$. It is not practical at this stage because using the free hole band inevitably introduced some error into our result. Furthermore, there are other factors such as orbital ordering which can influence $T_{\mathrm{C}}$ are not considered here.

In summary, we used the $(2 S+1)$ expansion method to calculate the magnetization and $T_{\mathrm{C}}$ of a DE system. The $T_{\mathrm{C}}$ of a pure DE system is about twice of the experimental result. When SE is incorporated, the Curie temperature is suppressed to realistic value. Therefore, we can dispense with the doubt on whether $\mathrm{DE}$ is the fundamental mechanism in manganites.

This work is supported in part by the National Science Council, Taiwan ROC under the contract number NSC 88-2112-M-002-007.

\section{Appendix}

Here we illustrate the method of calculation. The calculation involving spin operators are quite complicated because, unlike those of creation and annihilation operators, the commutators of them are operators. The fact that we wanted to work in finite-temperature range makes the calculation more tedious. We begin by giving some simple results of $H_{0}$ in which $s_{z}$ is a good quantum number:

$$
\left[H_{0}, S_{z}\right]=0
$$




$$
\left[H_{0}, S_{ \pm}\right]=\mp \omega_{0} S_{+} .
$$

and

$$
S_{ \pm}(\tau)=\mathrm{e}^{\mp \tau \omega_{0}} S_{ \pm}
$$

Consider the evaluation of $\left\langle\hat{T} S_{+}(\tau) S_{-}\right\rangle$where $\hat{T}$ is the time-order operator:

$$
\begin{aligned}
\left\langle\hat{T} S_{+}(\tau) S_{-}\right\rangle & =\theta(\tau)\left\langle S_{+}(\tau) S_{-}\right\rangle+\theta(-\tau)\left\langle S_{-} S_{+}(\tau)\right\rangle \\
& =\sum_{n} \mathrm{e}^{-\beta E_{n}}\left[\theta(\tau)\left\langle n\left|S_{+} S_{-}\right| n\right\rangle\right.
\end{aligned}
$$

$$
\left.+\theta(-\tau)\left\langle n\left|S_{-} S_{+}\right| n\right\rangle\right] \mathrm{e}^{-\omega_{0} \tau},
$$

where $E_{n}$ is the energy of state $|n\rangle$. Taking its Fourier transform as that in the Matsubara formalism, we get

$$
\begin{aligned}
D_{0}\left(\mathrm{i} q_{n}\right) & =-\frac{1}{2} \int_{-\beta}^{\beta} \mathrm{d} \tau\left\langle\hat{T} S_{+}(\tau) S_{-}\right\rangle \mathrm{e}^{\mathrm{i} q_{n} \tau} \\
& =\frac{2\left\langle S_{z}\right\rangle}{\mathrm{i} q_{n}-\omega_{0}} .
\end{aligned}
$$

Now consider $\left\langle\hat{T} S_{z}\left(\tau_{1}\right) S_{+}(\tau) S_{-}\left(\tau^{\prime}\right)\right\rangle$ :

$$
\begin{aligned}
& \left\langle\hat{T} S_{z}\left(\tau_{1}\right) S_{+}(\tau) S_{-}\left(\tau^{\prime}\right)\right\rangle \\
& \quad=\theta\left(\tau_{1}-\tau\right) \theta\left(\tau-\tau^{\prime}\right)\left\langle S_{z} S_{+}(\tau) S_{-}\left(\tau^{\prime}\right)\right\rangle+\theta\left(\tau_{1}-\tau^{\prime}\right) \theta\left(\tau^{\prime}-\tau\right)\left\langle S_{z} S_{-}\left(\tau^{\prime}\right) S_{+}(\tau)\right\rangle \\
& \quad+\theta\left(\tau-\tau_{1}\right) \theta\left(\tau_{1}-\tau^{\prime}\right)\left\langle S_{+}(\tau) S_{z} S_{-}\left(\tau^{\prime}\right)\right\rangle+\theta\left(\tau-\tau^{\prime}\right) \theta\left(\tau^{\prime}-\tau_{1}\right)\left\langle S_{+}(\tau) S_{-}\left(\tau^{\prime}\right) S_{z}\right\rangle \\
& \quad+\theta\left(\tau^{\prime}-\tau_{1}\right) \theta\left(\tau_{1}-\tau\right)\left\langle S_{-}\left(\tau^{\prime}\right) S_{z} S_{+}(\tau)\right\rangle+\theta\left(\tau^{\prime}-\tau\right) \theta\left(\tau-\tau_{1}\right)\left\langle S_{-}\left(\tau^{\prime}\right) S_{+}(\tau) S_{z}\right\rangle
\end{aligned}
$$

In view of eqs. (A-1) and (A.3), we can reduce, for example, the third term in eq. (A.6) as thus: (the step function is not shown):

$$
\begin{aligned}
& \left\langle S_{+}(\tau) S_{z} S_{-}\left(\tau^{\prime}\right)\right\rangle \\
& \quad=\mathrm{e}^{\omega_{0}\left(\tau^{\prime}-\tau\right)}\left\langle S_{+} S_{z} S_{-}\right\rangle \\
& \quad=\mathrm{e}^{\omega_{0}\left(\tau^{\prime}-\tau\right)}\left[\left\langle\left[S_{+}, S_{z}\right] S_{-}\right\rangle+\left\langle S_{z} S_{+} S_{-}\right\rangle\right] \\
& \quad=\mathrm{e}^{\omega_{0}\left(\tau^{\prime}-\tau\right)}\left[-\left\langle S_{+} S_{-}\right\rangle+\left\langle S_{z}\left[S_{+}, S_{-}\right]\right\rangle+\left\langle S_{z} S_{-} S_{+}\right\rangle\right] .
\end{aligned}
$$

Next, using the invariance of trace under cyclic rotation, we can change the last term of eq. (A.7) into the original term, keeping in mind that there is an ensemble average operator $\exp \left(-\beta H_{0}\right)$ :

$$
\begin{aligned}
\left\langle S_{+}(\tau) S_{z} S_{-}\left(\tau^{\prime}\right)\right\rangle= & \mathrm{e}^{\omega_{0}\left(\tau^{\prime}-\tau\right)}\left[-\left\langle S_{+} S_{-}\right\rangle+\left\langle S_{z}\left[S_{+}, S_{-}\right]\right\rangle\right. \\
& \left.+\mathrm{e}^{-\beta \omega_{0}}\left\langle S_{+} S_{z} S_{-}\right\rangle\right] \\
= & \frac{\mathrm{e}^{\omega_{0}\left(\tau-\tau^{\prime}\right)}}{1-\mathrm{e}^{-\beta \omega_{0}}}\left[-\left\langle S_{+} S_{-}\right\rangle+2\left\langle S_{z}^{2}\right\rangle\right]
\end{aligned}
$$

Now both terms can be evaluated in the ensemble of $H_{0}$. Clearly, our strategy is to convert the operators in the bracket to $S_{z}$ by moving them in a cyclic way and using commutation relations. Similar method had been used in refs. 19 and 22-24. All the six terms in eq. (A.6) can be computed the same way. As a result we have

$$
\begin{aligned}
\left\langle\hat{T} S_{z}\left(\tau_{1}\right) S_{+}(\tau) S_{-}\left(\tau^{\prime}\right)\right\rangle & \\
= & 2\left\langle S_{z}^{2}\right\rangle \mathrm{e}^{\omega_{0}\left(\tau^{\prime}-\tau\right)}\left[b_{0} \theta\left(\tau^{\prime}-\tau\right)+\left(b_{0}+1\right) \theta\left(\tau-\tau^{\prime}\right)\right] \\
& -2\left\langle S_{z}\right\rangle \mathrm{e}^{\omega_{0}\left(\tau^{\prime}-\tau\right)}\left[b_{0}\left(b_{0}+1\right)-b_{0} \theta\left(\tau^{\prime}-\tau_{1}\right) \theta\left(\tau_{1}-\tau^{\prime}\right)\right. \\
& \left.+\left(b_{0}+1\right) \theta\left(\tau^{\prime}-\tau_{1}\right) \theta\left(\tau_{1}-\tau\right)\right],
\end{aligned}
$$

where

$$
b_{0}=\frac{1}{\mathrm{e}^{\beta \omega_{0}}-1},
$$

Its Fourier transform is

$$
\begin{array}{r}
\int_{0}^{\beta} \mathrm{d} \tau \int_{0}^{\beta} \mathrm{d} \tau_{1} \int_{0}^{\beta} \mathrm{d} \tau^{\prime}\left\langle\hat{T} S_{+}(\tau) S_{z}\left(\tau_{1}\right) S_{-}\left(\tau^{\prime}\right)\right\rangle \mathrm{e}^{\mathrm{i}\left(q_{l} \tau+q_{m} \tau_{1}+q_{n} \tau^{\prime}\right)} \\
=-\frac{2 \beta^{2}\left\langle S_{z}^{2}\right\rangle \delta_{0, q_{m}} \delta_{0, q_{l}+q_{n}}}{\mathrm{i} q_{n}-\omega_{0}}+\frac{2 \beta\left\langle S_{z}\right\rangle\left(\delta_{0, q_{m}}+\delta_{0, q_{l}+q_{m}+q_{n}}\right)}{\left(\mathrm{i} q_{l}-\omega_{0}\right)\left(\mathrm{i} q_{n}+\omega_{0}\right)}
\end{array}
$$

Here, our result is different from that of ref. 19. They might have neglected the last two terms of eq. (A.9) which have abnormal time-dependence and give the term $\delta_{0, q_{m}}$ in the bracket of the second term.

We may also encounter $\left\langle\hat{T} S_{+}\left(\tau_{1}\right) S_{+}\left(\tau_{2}\right) S_{-}\left(\tau_{3}\right) S_{-}\left(\tau_{4}\right)\right\rangle$. The second-order terms of $H_{4}$ contain this factor. Like that in eq. (A.6), we can decompose it into 24 terms due to the time arguements of the spin operators. A typical term is

$\theta\left(\tau_{1}-\tau_{2}\right) \theta\left(\tau_{2}-\tau_{3}\right) \theta\left(\tau_{3}-\tau_{4}\right) \mathrm{e}^{\omega_{0}\left(\tau_{2}-\tau_{1}+\tau_{4}-\tau_{3}\right)}\left\langle S_{+} S_{-} S_{+} S_{-}\right\rangle$, where the order of the spin operators are fixed. To keep track of the operator, we added a subscript to each of them. By moving $S_{+1}$ in a cyclic way, we get

$$
\begin{aligned}
& \left\langle S_{+1} S_{+2} S_{-3} S_{-4}\right\rangle \\
& \quad=2\left\langle S_{+2} S_{z} S_{-4}\right\rangle+2\left\langle S_{+2} S_{-3} S_{z}\right\rangle+\left\langle S_{+2} S_{-3} S_{-4} S_{+1}\right\rangle \\
& \quad=\frac{1}{1-\mathrm{e}^{-\beta \omega_{0}}}\left[2\left\langle S_{+} S_{z} S_{-}\right\rangle+2\left\langle S_{+} S_{-} S_{z}\right\rangle\right]
\end{aligned}
$$

The resulting terms can be further reduced in the manner of eqs. (A.7) and (A.8). Other terms can also be reduced the same way. As a result, 


$$
\begin{aligned}
\left\langle\hat{T} S_{+}\right. & \left.\left(\tau_{1}\right) S_{+}\left(\tau_{2}\right) S_{-}\left(\tau_{3}\right) S_{-}\left(\tau_{4}\right)\right\rangle \\
= & \left\langle S_{z}^{2}\right\rangle\left[\mathcal{D}\left(\tau_{1}-\tau_{3}\right) \mathcal{D}\left(\tau_{2}-\tau_{4}\right)+\mathcal{D}\left(\tau_{1}-\tau_{4}\right) \mathcal{D}\left(\tau_{2}-\tau_{3}\right)\right] \\
& -2\left\langle S_{z}\right\rangle \operatorname{coth} \beta \omega_{0}\left[\mathcal{D}\left(\tau_{1}-\tau_{3}\right) \mathcal{D}\left(\tau_{2}-\tau_{4}\right)+\mathcal{D}\left(\tau_{1}-\tau_{4}\right) \mathcal{D}\left(\tau_{2}-\tau_{3}\right)\right] \\
& +2\left\langle S_{z}\right\rangle\left\{b _ { 0 } \left[\xi\left(\tau_{3}, \tau_{1}, \tau_{4}, \tau_{2}\right)+\xi\left(\tau_{3}, \tau_{2}, \tau_{4}, \tau_{1}\right)+\xi\left(\tau_{4}, \tau_{1}, \tau_{3}, \tau_{2}\right)\right.\right. \\
& \left.+\xi\left(\tau_{4}, \tau_{2}, \tau_{3}, \tau_{1}\right)\right]+\left(b_{0}+1\right)\left[\xi\left(\tau_{1}, \tau_{3}, \tau_{2}, \tau_{4}\right)+\xi\left(\tau_{1}, \tau_{4}, \tau_{2}, \tau_{3}\right)\right. \\
& \left.\left.+\xi\left(\tau_{2}, \tau_{3}, \tau_{1}, \tau_{4}\right)+\xi\left(\tau_{2}, \tau_{4}, \tau_{1}, \tau_{3}\right)\right]\right\} \mathrm{e}^{\omega_{0}\left(\tau_{3}+\tau_{4}-\tau_{1}-\tau_{2}\right)}
\end{aligned}
$$

where

$$
\xi\left(\tau_{1}, \tau_{2}, \tau_{3}, \tau_{4}\right)=\theta\left(\tau_{1}-\tau_{2}\right) \theta\left(\tau_{2}-\tau_{3}\right) \theta\left(\tau_{3}-\tau_{4}\right),
$$

and

$$
\mathcal{D}\left(\tau_{1}-\tau_{2}\right)=2\left[b_{0} \theta\left(\tau_{2}-\tau_{1}\right)+\left(b_{0}+1\right) \theta\left(\tau_{1}-\tau_{2}\right)\right] \mathrm{e}^{\omega_{0}\left(\tau_{2}-\tau_{1}\right)} .
$$

Finally, we need to calculate $\left\langle\hat{T} S_{+}(\tau) S_{-}\left(\tau^{\prime}\right) S_{z}\left(\tau_{1}\right) S_{z}\left(\tau_{2}\right)\right\rangle$. As before, the order of time gives us 24 terms. All of them can be computed the same way. For example,

$$
\begin{aligned}
& \left\langle S_{+}(\tau) S_{-}\left(\tau^{\prime}\right) S_{z}\left(\tau_{1}\right) S_{z}\left(\tau_{2}\right)\right\rangle \\
& \quad=\frac{\mathrm{e}^{\omega_{0}\left(\tau_{2}-\tau_{1}\right)}}{1-\mathrm{e}^{-\beta \omega_{0}}}\left(2\left\langle S_{z}^{3}\right\rangle-\left\langle S_{-} S_{+} S_{z}\right\rangle-\left\langle S_{-} S_{z} S_{+}\right\rangle\right) \\
& \quad=\mathrm{e}^{\omega_{0}\left(\tau-\tau^{\prime}\right)}\left[\frac{2\left\langle S_{z}^{3}\right\rangle}{1-\mathrm{e}^{-\beta \omega_{0}}}-\frac{4 \mathrm{e}^{-\beta \omega_{0}}\left\langle S_{z}^{2}\right\rangle}{\left(1-\mathrm{e}^{-\beta \omega_{0}}\right)^{2}}+\frac{4 \mathrm{e}^{-2 \beta \omega_{0}}\left\langle S_{z}\right\rangle}{\left(1-\mathrm{e}^{-\beta \omega_{0}}\right)^{3}}\right] .
\end{aligned}
$$

As a result,

$$
\begin{aligned}
\left\langle\hat{T} S_{+}\right. & \left.(\tau) S_{-}\left(\tau^{\prime}\right) S_{z}\left(\tau_{1}\right) S_{z}\left(\tau_{2}\right)\right\rangle \\
= & \left\langle S_{z}^{3}\right\rangle \mathcal{D}\left(\tau-\tau^{\prime}\right)+2\left\langle S_{z}^{2}\right\rangle\left\{b _ { 0 } \left[2 \xi\left(\tau^{\prime}, \tau_{1}, \tau_{2}, \tau\right)+2 \xi\left(\tau^{\prime}, \tau_{2}, \tau_{1}, \tau\right)\right.\right. \\
& \left.+\xi\left(\tau^{\prime}, \tau_{2}, \tau, \tau_{1}\right)+\xi\left(\tau^{\prime}, \tau_{1}, \tau, \tau_{2}\right)+\xi\left(\tau_{1}, \tau^{\prime}, \tau_{2}, \tau\right)+\xi\left(\tau_{2}, \tau^{\prime}, \tau_{1}, \tau\right)\right] \\
& -\left(b_{0}+1\right)\left[2 \xi\left(\tau, \tau_{1}, \tau_{2}, \tau^{\prime}\right)+2 \xi\left(\tau, \tau_{2}, \tau_{1}, \tau^{\prime}\right)+\xi\left(\tau, \tau_{1}, \tau^{\prime}, \tau_{2}\right)\right. \\
& \left.\left.+\xi\left(\tau, \tau_{2}, \tau^{\prime}, \tau_{1}\right)+\xi\left(\tau_{1}, \tau, \tau_{2}, \tau^{\prime}\right)+\xi\left(\tau_{2}, \tau, \tau_{1}, \tau^{\prime}\right)\right]-2 b_{0}\left(b_{0}+1\right)\right\} \\
& +2\left\langle S_{z}\right\rangle\left\{2 b_{0}\left(b_{0}+1\right)\left[2\left(b_{0}+1\right) \theta\left(\tau-\tau^{\prime}\right)+2 b_{0} \theta\left(\tau^{\prime}-\tau\right)\right]\right. \\
& +\left(b_{0}+1\right)^{2}\left[\xi\left(\tau, \tau_{1}, \tau_{2}, \tau^{\prime}\right)+\xi\left(\tau, \tau_{2}, \tau_{1}, \tau^{\prime}\right)\right] \\
& -b_{0}{ }^{2}\left[\xi\left(\tau^{\prime}, \tau_{1}, \tau_{2}, \tau\right)+\xi\left(\tau^{\prime}, \tau_{2}, \tau_{1}, \tau\right)\right] \\
& +b_{0}\left(b_{0}+1\right)\left[\xi\left(\tau^{\prime}, \tau, \tau_{1}, \tau_{2}\right)+\xi\left(\tau_{1}, \tau_{2}, \tau^{\prime}, \tau\right)+\xi\left(\tau_{1}, \tau^{\prime}, \tau, \tau_{2}\right)\right. \\
& \left.-\xi\left(\tau, \tau^{\prime}, \tau_{1}, \tau_{2}\right)-\xi\left(\tau_{1}, \tau_{2}, \tau, \tau^{\prime}\right)-\xi\left(\tau_{1}, \tau, \tau^{\prime}, \tau_{2}\right)+(1 \leftrightarrow 2)\right\}
\end{aligned}
$$

There seems no simple rules to obtain all the terms.

1) For a recent review on experimental results, see A. P. Ramirez: J. Phys. C.: Condens. Matter 9 (1997) 8171.

2) C. Zener: Phys. Rev. 82 (1951) 403.

3) A. J. Millis, P. B. Littlewood and B. I. Shraiman: Phys. Rev. Lett. 74 (1995) 5144.

4) J. Millis, Boris I. Shraiman and R. Mueller: Phys. Rev. Lett. 77 (1996) 175.

5) G. Jacob, W. Westerburg, F. Martin and H. Adrain: Phys. Rev. B 80 (1998) 14966

6) M. Jaime, M. B. Salarmon and K. Pettit: Appl. Phys. Lett. 68 (1996) 1576.

7) H. Röder, Jun Zang and A. R. Bishop: Phys. Rev. Lett. 76 (1996) 1356.

8) Priya Mahadevan, N. Shanthi and D. D. Sarma: Phys. Rev. B 54 (1996) 11199

9) Nobuo Furukawa: J. Phys. Soc. Jpn. 63 (1994) 3214.

10) C. M. Varma: Phys. Rev. B 54 (1996) 7328.

11) L. Sheng, D. Y. Xing, D. N. Sheng and C. S. Ting: Phys. Rev. Lett. 79 (1997) 1710
12) L. Sheng, D. N. Sheng and C. S. Ting: Phys. Rev. B 59 (1997) 13550.

13) P. W. Anderson and H. Hasegawa: Phys. Rev. 100 (1955) 675.

14) De Gennes: Phys. Rev. 118 (1960) 141

15) K. Kubo and N. Ohata: J. Phys. Soc. Jpn. 33 (1972) 21.

16) Sanjoy K. Sarker: J. Phys.: Condens. Matter 8 (1996) L515.

17) D. M. Edwards, A. C. M. Green and K. Kubo: J. Phys.: Condens. Matter 11 (1999) 2791.

18) T. Okabe: Prog. Theo. Phys. 97 (1997) 21.

19) V. G. Vaks, A. I. Larkin and S. A. Pikin: JETP 26 (1968) 188. However, some of our results are different.

20) We can not have the term $n_{1}=n$ and $n_{l \neq 1}=0$ because the holes have to hop to nearest neighbors.

21) This is the random-phase approximation, just like that in ref. 19. But our mean-field is different.

22) M. Gaudin: Nucl. Phys. 15 (1960) 89

23) B. Giovannini, M. Peter and S. Koide: Phys. Rev. 149 (1965) 251.

24) B. Giovannini and S. Koide: Prog. Theor. Phys. 34 (1965) 705 . 